\title{
Is the generalized Brink-Axel hypothesis valid?
}

\author{
M. Guttormsen ${ }^{* a}$, A. C. Larsen ${ }^{a}$, A. Görgen ${ }^{a}$, T. Renstrøm ${ }^{a}$, S. Siem ${ }^{a}$, T. G. Tornyi ${ }^{a}{ }^{b}$ \\ and G. M. Tveten ${ }^{a}$ \\ ${ }^{a}$ Department of Physics, University of Oslo, Norway \\ ${ }^{b}$ Department of Nuclear Physics, Australian National University, Canberra, Australia \\ E-mail: magne.guttormsen@fys.uio.no
}

\begin{abstract}
Experimental results of the ${ }^{237} \mathrm{~Np}(d, p \gamma)^{238} \mathrm{~Np}$ reaction are presented, which verifies the generalized Brink-Axel (gBA) hypothesis for $\gamma$ transitions between states in the quasi-continuum. The gBA hypothesis holds not only for specific collective resonances, but for the full dipole strength below the neutron separation energy. We discuss the validity of the gBA hypothesis also for lighter systems like ${ }^{92} \mathrm{Zr}$ where the concept of a unique $\gamma$-ray strength function $(\gamma \mathrm{SF})$ is problematic due to large Porter-Thomas fluctuations. Methods for studying the $\gamma \mathrm{SF}$ and the fluctuations as function of excitation energy are presented.
\end{abstract}

The 26th International Nuclear Physics Conference

11-16 September, 2016

Adelaide, Australia

${ }^{*}$ Speaker. 


\section{Introduction}

More than sixty years ago, Brink proposed in his doctoral thesis at Oxford University $[1,2]$ that the photoabsorption cross section of the giant electric dipole resonance (GDR) is independent of the detailed structure of the initial state. The hypothesis was further extended by the principle of detailed balance, to include absorption and emission of $\gamma$ rays between resonant states [3, 4]. In more general terms, this generalized Brink-Axel (gBA) hypothesis implies that the dipole $\gamma$-decay strength has no explicit dependence on excitation energy, spin or parity, except the obvious dipole transition selection rules.

The gBA hypothesis is frequently applied in a variety of applications as it dramatically simplifies the considered problem and in some cases, is a necessity in order to perform the calculations. Hence, the question of whether the hypothesis is valid or not, and under which circumstances, is of utmost importance. For this discussion, one should keep in mind that the original formulation of the hypothesis was meant for moderate energies, as cited from Brink's thesis: "Now we assume that the energy dependence of the photo effect is independent of the detailed structure of the initial state so that, if it were possible to perform the photo effect on an excited state, the cross section for absorption of a photon of energy $E$ would still have an energy dependence given by (15)", where the equation (15) refers to a Lorentzian shape of the photoabsorption cross section on the ground state.

The gBA hypothesis has fundamental impact on nuclear structure and dynamics, and has a pivotal role in the description of $\gamma$ and $\beta$ decay for applied nuclear physics. In particular, the hypothesis is often used for calculating $(n, \gamma)$ cross-section needed to model the astrophysical $r$ process nucleosynthesis and the next-generation of fast nuclear reactors.

Experimental and theoretical information on the validity of the gBA hypothesis is rather scarce. Primary transitions from $(n, \gamma)$ reactions give $\gamma$ SFs consistent with the gBA hypothesis, but only in a limited $\gamma$-energy range $[5,6,7,8,9]$. On the other hand, the ${ }^{89} \mathrm{Y}(p, \gamma)^{90} \mathrm{Zr}$ reaction points towards deviations from the gBA hypothesis [10]. There have also been theoretical attempts to test the gBA hypothesis. Here, deviations and even violations to the hypothesis are found $[11,12]$. However, for other theoretical applications, the assumption of the gBA hypothesis is successfully applied $[13,14,15,16,17]$.

From the above mentioned findings, it is not at all obvious when the gBA hypothesis is valid. This unclear situation is not only due to peculiar structures and dynamics of the systems studied, but also in some cases, due to huge Porter-Thomas fluctuations [18] that may camouflage the underlying physics. In this work, we will show that at moderate excitation energies and with a proper averaging over many $\gamma$ transitions, the gBA hypothesis is a fruitful concept for the exploration of the nuclear quasi-continuum region.

\section{Method and tools}

The Oslo method has proven to be a robust technique to extract gross properties in the energy region below the particle separation energy in terms of nuclear level density (NLD) and $\gamma$-ray strength function $(\gamma \mathrm{SF})[19,20]$. The method is based on measuring the $\gamma$ decay after light ion reactions where only one charged particle is ejected. In this way, one may collect $\gamma$ spectra tagged 


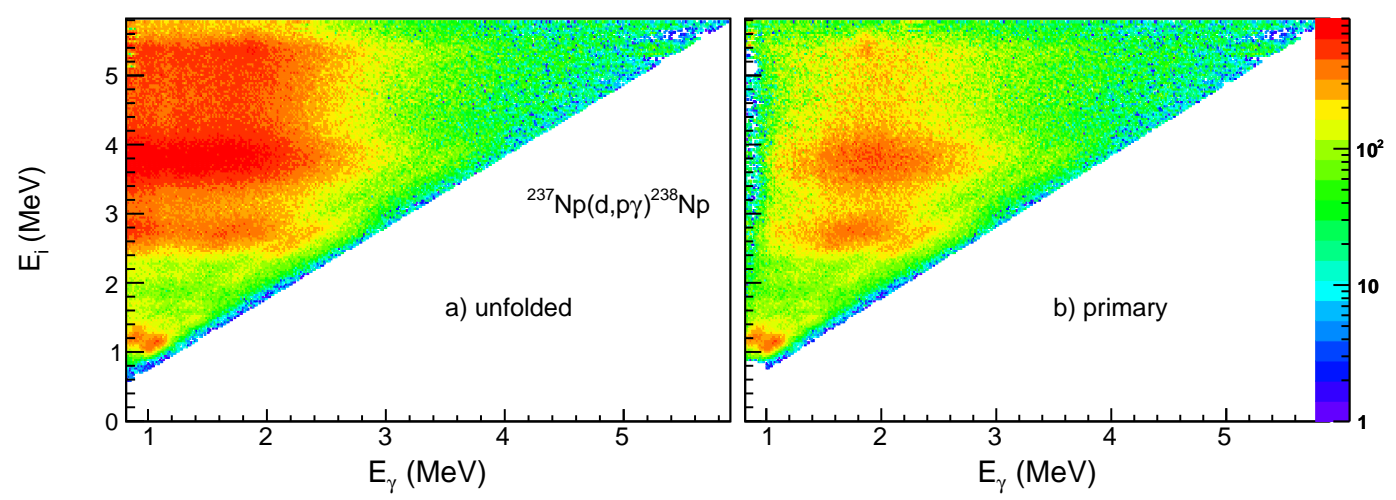

Figure 1: (Color online) Initial excitation energy $E_{i}$ versus $\gamma$-ray energy $E_{\gamma}$ from particle- $\gamma$ coincidences recorded with the ${ }^{237} \mathrm{~Np}(d, p \gamma){ }^{238} \mathrm{~Np}$ reaction [23, 24]. The $\gamma$-ray spectra unfolded by the NaI response function (a) and the primary or first-generation $\gamma$-ray spectra $P\left(E_{\gamma}, E_{i}\right)$ (b) are extracted as function of initial excitation energy $E_{i}$.

with the excitation energy. Such coincidence data are then organized into an $\left(E_{\gamma}, E_{x}\right)$ matrix, which represents the basis for the Oslo method. Without going into details, this matrix is then corrected for the detector response function [21] and then utilized to extract the first-generation (primary) $\gamma$ spectra [22]. For illustration, the unfolded and primary $\gamma$ matrices of ${ }^{238} \mathrm{~Np}$ are shown in Fig. 1

The primary $\gamma$-ray matrix, which represents the energy distribution of the first emitted $\gamma$-ray transition in each cascade (see Fig. 1 (b) ), is assumed to be factorized by

$$
P\left(E_{\gamma}, E_{i}\right) \propto \rho\left(E_{i}-E_{\gamma}\right) \mathscr{T}\left(E_{\gamma}\right) .
$$

The proportionality to $\rho\left(E_{i}-E_{\gamma}\right)$ is in accordance with the Fermi's golden rule [25, 26], and the second factor, the $\gamma$-ray transmission coefficient $\mathscr{T}$, is assumed to be independent of excitation energy according to the Brink hypothesis [1]. For dipole transitions, $\mathscr{T}$ relates to the $\gamma \mathrm{SF}$ by

$$
f\left(E_{\gamma}\right)=\frac{1}{2 \pi} \frac{\mathscr{T}\left(E_{\gamma}\right)}{E_{\gamma}^{3}}
$$

In the standard Oslo method, we utilize the $\gamma$ spectra above a certain initial excitation region (in the ${ }^{238} \mathrm{~Np}$ case, $E_{\min }=3.03 \mathrm{MeV}$ ) where the decay is assumed to be of statistical nature. The upper excitation region is limited to the neutron separation energy by $E_{\max } \approx S_{n}$.

By accepting the level density obtained with the fit of $\rho$ and $\mathscr{T}$ to a large part of the $P$ matrix (the standard Oslo method), we may investigate the transmission coefficient in more detail, and thus the validity of the Brink hypothesis. We adopt the solutions $\mathscr{T}$ and $\rho$ from Eq. (2.1) and write

$$
\mathscr{T}\left(E_{\gamma}, E_{i}\right) \approx \mathscr{N}\left(E_{i}\right) \frac{P\left(E_{\gamma}, E_{i}\right)}{\rho\left(E_{i}-E_{\gamma}\right)} .
$$

with a normalization function given by

$$
\mathscr{N}\left(E_{i}\right)=\frac{\int_{0}^{E_{i}} \mathrm{~d} E_{\gamma} \mathscr{T}\left(E_{\gamma}\right) \rho\left(E_{i}-E_{\gamma}\right)}{\int_{0}^{E_{i}} \mathrm{~d} E_{\gamma} P\left(E_{\gamma}, E_{i}\right)} .
$$




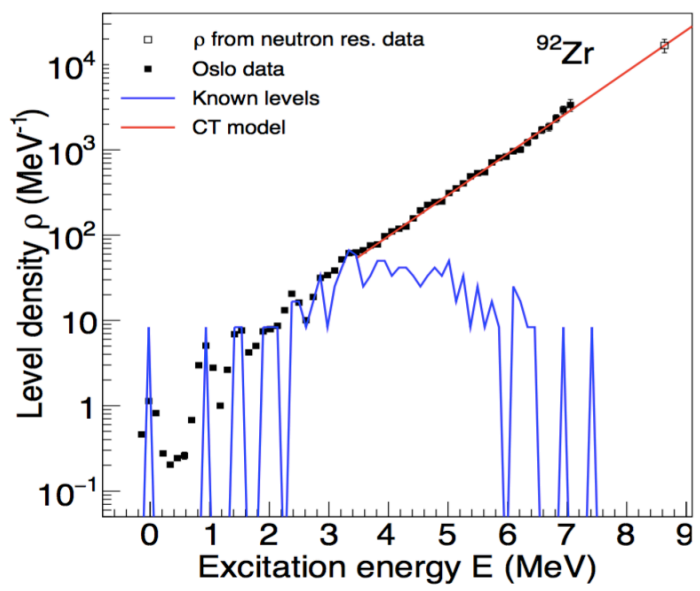

Six decay possibilities for dipole transitions

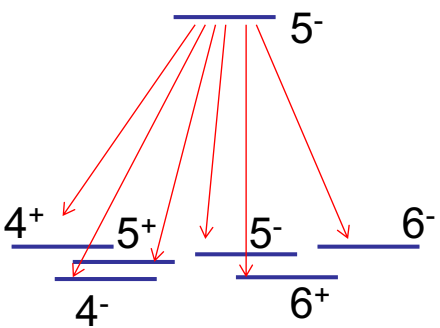

Figure 2: (Color online) The level density extracted from the ${ }^{92} \mathrm{Zr}\left(p, p^{\prime}\right)^{92} \mathrm{Zr}$ reaction based on the primary $P\left(E_{\gamma}, E_{i}\right)$ matrix. In general, the dipole transition in the quasi-continuum goes with six transitions: two stretched $M 1$, two stretched $E 1$, one un-stretched $M 1$ and one un-stretched $E 1$ transition.

Since $\mathscr{N}$ only depends on $E_{i}$, we may now study how $\mathscr{T}$ (and thus $f$ ) varies as function of $E_{\gamma}$ for each excitation bin. An expression for $\mathscr{T}\left(E_{\gamma}, E_{f}\right)$ can be constructed in the same way as above [24].

The fluctuations expected for the $\gamma \mathrm{SF}$ are assumed to follow the $\chi_{v}^{2}$ distribution where $v$ is the number of transitions $n$ included in the averaging for a certain energy $E_{\gamma}$. The relative fluctuations of the $\chi_{v}^{2}$ distribution are given by $r=\sqrt{2 / v}$. With the experimental information on the level density $\rho$ (see e.g. Fig. 2), we may count the number of transitions expected from an initial to a final excitation energy bin. For the standard Oslo method, a large part of the primary $\gamma$ matrix is utilized to obtain a good averaging. Here, all the rows from $E_{\min }$ to $E_{\max }$ of the $P$ matrix are included:

$$
n\left(E_{\gamma}\right)=\Delta E^{2} \sum_{E_{i}=E_{\min }}^{E_{\max }} \sum_{I \pi} \sum_{L=-1}^{1} \sum_{\pi^{\prime}} \rho\left(E_{i}, I, \pi\right) \cdot \rho\left(E_{i}-E_{\gamma}, I+L, \pi^{\prime}\right),
$$

where $\Delta E$ is the energy-bin width for initial and final excitation energies. If we assume the decay from only one initial excitation energy bin, the number of transitions is given by

$$
n\left(E_{\gamma}, E_{i}\right)=\Delta E^{2} \sum_{I \pi} \sum_{L=-1}^{1} \sum_{\pi^{\prime}} \rho\left(E_{i}, I, \pi\right) \cdot \rho\left(E_{i}-E_{\gamma}, I+L, \pi^{\prime}\right) .
$$

A similar expression can be constructed for the cases when the decay ends at a certain final excitation energy $E_{f}=E_{i}-E_{\gamma}$. The two formulae above have to be taken with care if not all spins and parities are available. In particular, for the direct dipole decay to the $0^{+}$ground state, only one 

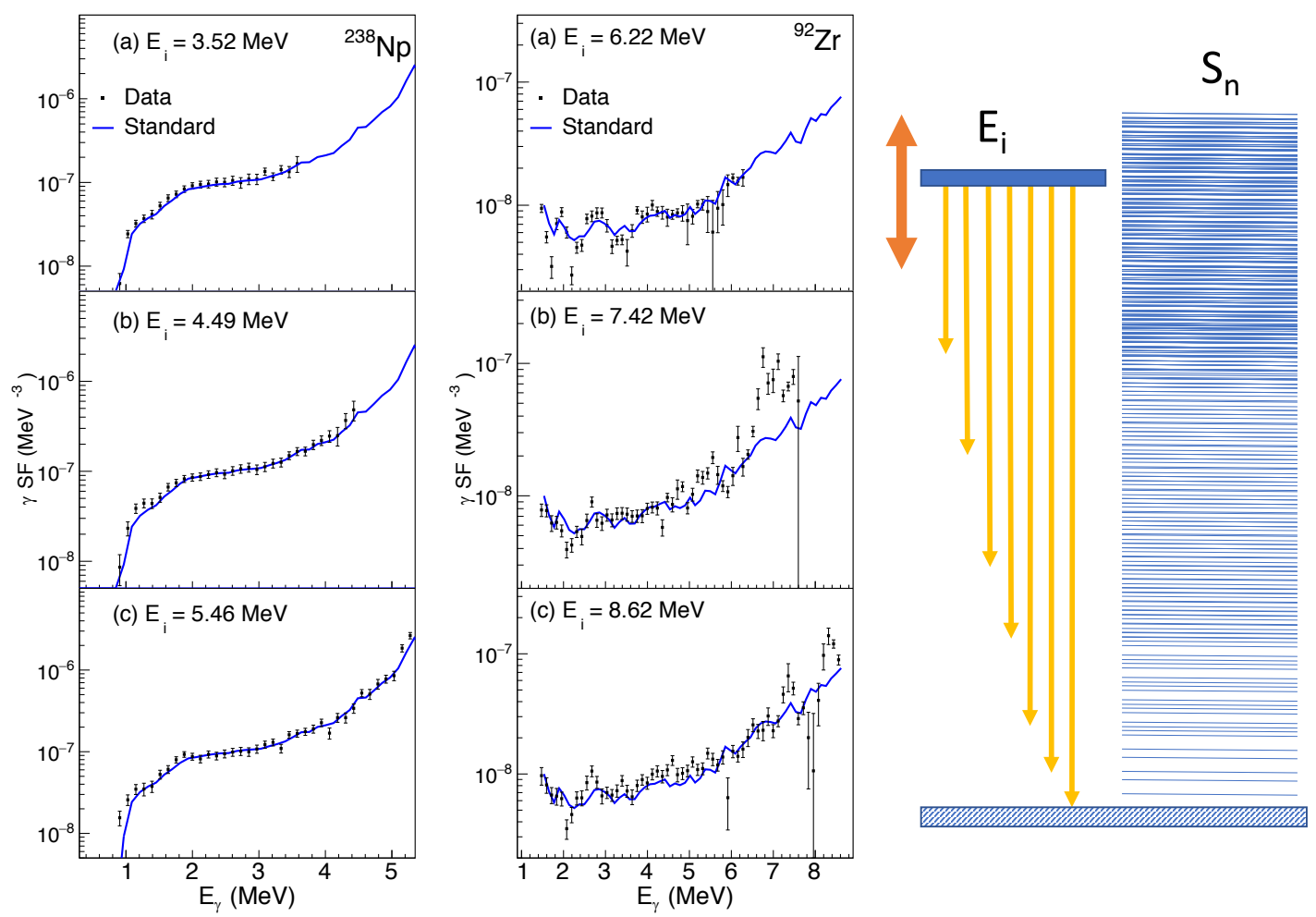

Figure 3: (Color online) Gamma-ray strength functions of ${ }^{238} \mathrm{~Np}$ (left) and ${ }^{92} \mathrm{Zr}$ (right) extracted from various initial excitation bins $E_{i}$ (see illustration). The data points with error bars are from statistically independent data sets. The blue lines are obtained by the standard Oslo method where a large part of the primary $\gamma$-ray matrix has been exploited giving a better averaging of the $\gamma$ SF by including more transitions. We observe significant fluctuations in the ${ }^{92} \mathrm{Zr}$ case.

transition appears, either one stretched $M 1$ or $E 1$ transition. As illustrated in Fig. 2, this is contrary to six transitions in the high level density region.

\section{Discussion}

In the following we will discuss the $\gamma$ decay in the quasi-continuum of ${ }^{238} \mathrm{~Np}$ and ${ }^{92} \mathrm{Zr}$. Both nuclei have been measured with high statistics allowing us to draw conclusions on the dependence of the $\gamma \mathrm{SF}$ s with excitation energy. The odd-odd ${ }^{238} \mathrm{~Np}$ nucleus has an extremely high level density of $\approx 43$ million levels per $\mathrm{MeV}$ at $S_{n}=5.488 \mathrm{MeV}$. Also at low excitation energy of $\approx 200 \mathrm{keV}$ we find $\approx 200$ levels per MeV. Thus, for this ultimate system, we do not expect significant PorterThomas fluctuations and the gBA hypothesis can be tested. However, the ${ }^{92} \mathrm{Zr}$ nucleus represents a system with $\approx 10000$ times less levels than ${ }^{238} \mathrm{~Np}$ making it more difficult to draw conclusions on the validity of the gBA hypothesis.

Figure 3 shows $\gamma \mathrm{SF}$ from three initial excitation bins by use of Eq. (2.3). The blue curve is based on the standard Oslo method of Eq. (2.1) including many more primary $\gamma$ transitions. In the case of ${ }^{238} \mathrm{~Np}$, the individual $\gamma \mathrm{SF}$ s indeed follow the same function obtained with the standard Oslo 

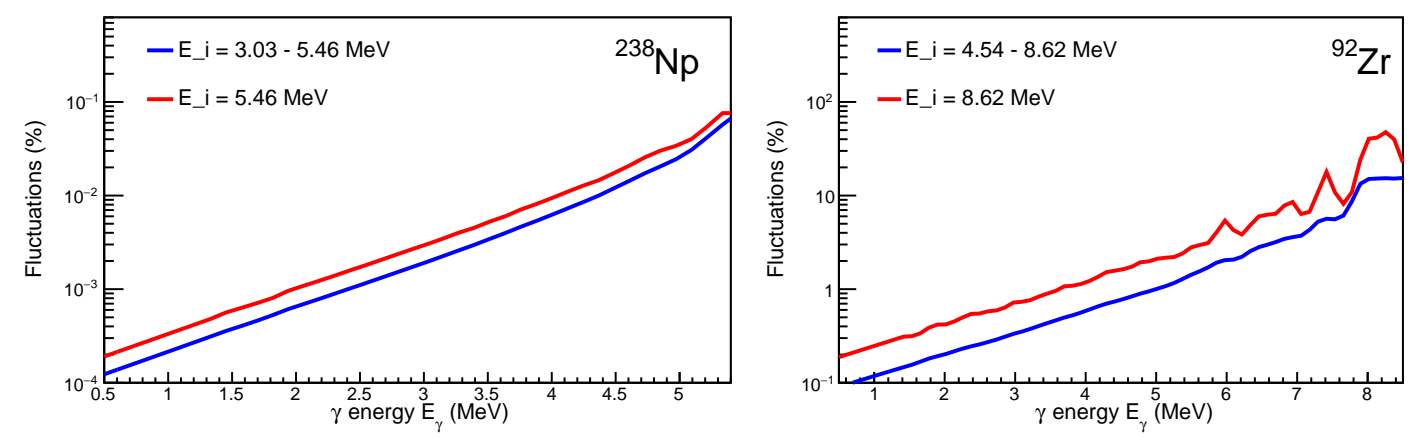

Figure 4: (Color online) Relative fluctuations $r\left(E_{\gamma}\right)$ in the $\gamma$ strength of ${ }^{238} \mathrm{~Np}$ (left) and ${ }^{92} \mathrm{Zr}$ (right) as function of $E_{\gamma}$. The red lines are evaluated for a narrow initial high-energy excitation bin, and the blue curves are the results based on a broad initial excitation region (standard Oslo method).

method. The close resemblance with a constant excitation-independent $\gamma \mathrm{SF}$ for these three initial excitations is found for all the 22 excitation bins between 3.03 and $5.46 \mathrm{MeV}$ (not shown).

As expected, the ${ }^{92} \mathrm{Zr}$ nucleus reveals strong fluctuations where the individual $\gamma$ SFs deviate more from the standard Oslo method predictions (blue curve) than given by the statistical uncertainties. Since this nucleus has lower NLD, we even find for certain $E_{\gamma}$-values that none or very few $\gamma$ transitions exist within the given initial excitation gate $E_{i}$. As an example, in the right lower panel (c) of Figure 3 a drop is seen in the $\gamma \mathrm{SFs}$ for $E_{\gamma} \approx 8.0 \mathrm{MeV}$ since no level exists in ${ }^{92} \mathrm{Zr}$ at a final excitation energy of $E_{f}=E_{i}-E_{\gamma}=8.62-8.00 \mathrm{MeV}=0.62 \mathrm{MeV}$.

The findings of ${ }^{92} \mathrm{Zr}$ versus ${ }^{238} \mathrm{~Np}$ raise a need for a more quantitative prediction of the PorterThomas fluctuations. If there is an insufficient averaging of the number of $\gamma$ transitions, the fluctuations could be large and the individual $\gamma$ SFs should then be different. However, this may not invalidate the gBA hypothesis, which predicts an underlying directive for the $\gamma$-decay probabillity.

In order to estimate the Porter-Thomas fluctuations, we apply the expressions for the number of transitions described for the standard Oslo method in Eq. (2.5) and for a specific initial excitation energy in Eq. (2.6). The relative fluctuations $r\left(E_{\gamma}\right)$ of Fig. 4 are seen to increase exponentially with $E_{\gamma}$ for both nuclei. We also note that the relative fluctuations are 2 - 3 times less for the standard Oslo method compared to the fluctuations using an individual narrow initial excitation bin. The fluctuations in ${ }^{238} \mathrm{~Np}$ are extremely low going from $r \approx 0.0001 \%$ at low $E_{\gamma}$ and up to $\approx 0.1 \%$ at $S_{n}$. The ${ }^{92} \mathrm{Zr}$ nucleus shows higher fluctuations than the statistical errors, and up to $r \approx 40 \%$ at $E_{\gamma} \approx 8 \mathrm{MeV}$. The individual $\gamma \mathrm{SFs}$ of ${ }^{92} \mathrm{Zr}$ (only 3 out of 34 shown in Fig. 3) fluctuate around the $\gamma \mathrm{SFs}$ obtained with the standard Oslo method, and thus support the validity of the gBA hypothesis also for this light nucleus.

\section{Conclusion}

We have studied the $\gamma \mathrm{SF}$ between excitation energy bins in ${ }^{238} \mathrm{~Np}$ and ${ }^{92} \mathrm{Zr}$. The $\gamma$ decay in ${ }^{238} \mathrm{~Np}$ is independent on the excitation energy, and thus validates the gBA hypothesis. In this case, 
the hypothesis works not only for specific collective resonances, but for the whole dipole $\gamma \mathrm{SF}$ below the neutron separation energy.

A necessity for testing the validity of the gBA hypothesis is that the Porter-Thomas fluctuations are smaller than the experimental errors. This means that the sampling of the $\gamma \mathrm{SF}$ should include sufficiently many $\gamma$ transitions. A technique has been developed using the measured level density to estimate the number of transitions with a certain $\gamma$ energy.

For the case of ${ }^{92} \mathrm{Zr}$, large fluctuations are found that apparently contradict the occurence of one unique $\gamma \mathrm{SF}$ for all excitation energies. A closer examination reveals that these large deviations appear due to few or non-existing levels for a given $E_{\gamma}$ at certain excitation bins. In particular, huge fluctuations appear for $\gamma$ SFs including low-lying states, e.g. the $I^{\pi}=0^{+}$ground state. The individual $\gamma \mathrm{SF}$ s seem to fluctuate around a common $\gamma \mathrm{SF}$, which gives support to the gBA hypothesis also for lighter nuclei.

\section{Acknowledgements}

We would like to thank E. A. Olsen, J. C. Müller, A. Semchenkov, and J. C. Wikne for providing high quality experimental conditions. A. C. L. gratefully acknowledges funding through ERCSTG-2014 under grant agreement no. 637686. G. M. T. gratefully acknowledges funding of this research from the Research Council of Norway, Project Grant No. 222287.

\section{References}

[1] D.M. Brink, Doctoral thesis, Oxford University, 1955.

[2] D.M. Brink 2009, available online at http://tid.uio.no/workshop09/talks/Brink.pdf

[3] P. Axel, Phys. Rev. 126, 671 (1962).

[4] G.A. Bartholomew, E.D. Earle, A.J. Ferguson, J.W. Knowles, and M.A. Lone, Adv. Nucl. Phys. 7, 229 (1973).

[5] M. Stefanon and F. Corvi, Nucl. Phys. A 281, 240 (1977).

[6] S. Raman, O. Shahal, and G.G. Slaughter, Phys. Rev. C 23, 2794 (1981).

[7] S. Kahane, S. Raman, G.G. Slaughter, C.Coceva and M. Stefanon, Phys. Rev. C 30, 807 (1984).

[8] M.A. Islam, T.J. Kennett, and W.V. Prestwich, Phys. Rev. C 43, 1086 (1990).

[9] J. Kopecky and M. Uhl, Phys. Rev. C 41, 1941 (1990).

[10] L. Netterdon, A. Endres, S. Goriely, J. Mayer, P. Scholz, M. Spieker, and A. Zilges, Phys. Lett. B 744, 358 (2015).

[11] C.W. Johnson, Phys. Lett. B 750, 72 (2015).

[12] G. Wendell Misch, George M. Fuller, and B. Alex Brown, Phys. Rev. C 90, 065808 (2014).

[13] T. Koeling, Nucl. Phys. A 307, 139 (1978).

[14] A. Höring and H.A. Weidenmüller, Phys. Rev. C 46, 2476 (1992).

[15] J.Z. Gu, H.A. Weidenmüller, Nucl. Phys. A 690, 382 (2001).

[16] E. Běták, F. Cvelbar, A. Likar, and T. Vidmar, Nucl. Phys. A 686, 204 (2001). 
[17] M.S. Hussein, B.V. Carlson and L.F. Canto, Nucl. Phys. A 731, 163 (2004).

[18] C.E. Porter and R.G. Thomas, Phys. Rev. 104, 483 (1956).

[19] A. Schiller, L. Bergholt, M. Guttormsen, E. Melby, J. Rekstad, and S. Siem, Nucl. Instrum. Methods Phys. Res. A 447, 494 (2000).

[20] A.C. Larsen et al., Phys. Rev. C 83, 034315 (2011).

[21] M. Guttormsen, T. S. Tveter, L. Bergholt, F. Ingebretsen, and J. Rekstad, Nucl. Instrum. Methods Phys. Res. A 374, 371 (1996).

[22] M. Guttormsen, T. Ramsøy, and J. Rekstad, Nucl. Instrum. Methods Phys. Res. A 255, 518 (1987).

[23] T.G. Tornyi et al., Phys. Rev. C 89, 0443232 (2014).

[24] M. Guttormsen, A. C. Larsen, A. Görgen, T. Renstrøm, S. Siem, T. G. Tornyi and G. M. Tveten, Phys. Rev. Lett. 116, 012502 (2016).

[25] P.A.M. Dirac, "The Quantum Theory of Emission and Absortion of Radiation". Proc. R. Soc. Lond. A 1927 114, 243-265.

[26] E. Fermi, Nuclear Physics. University of Chicago Press (1950). 